\title{
Subjective Evaluation of Specular Appearance for Multiple Observations Using Projector-Based Appearance Reproduction
}

\author{
Mayu Yokoya ${ }^{1}$, Shoji Yamamoto ${ }^{2}$, Yasuki Yamauchi ${ }^{3}$, Satoshi Yamamoto $^{4}$, \\ Osama Ouda ${ }^{5}$, Toshiya Nakaguchi ${ }^{6}$, and Norimichi Tsumura ${ }^{1,5}$ \\ 1 Department of Information \& Image Science, Chiba University, Japan \\ z7t0732@students.chiba-u.jp \\ 2 Tokyo Metropolitan College of Industrial Technology, Japan \\ ${ }^{3}$ Graduate School of Yamagata University, Japan \\ 4 Graduate School of Medicine, Chiba University, Japan \\ ${ }^{5}$ Graduate School of Advanced Integration Science, Chiba University, Japan \\ ${ }^{6}$ Graduate School of Engineering, Chiba University, Japan
}

\begin{abstract}
This paper presents the subjective evaluations of multiple observers as part of an investigation into the relationship between CG parameters of digital mockups and visual sensibility. In our experiments, the specular appearance of CG imagery is reproduced on an actual mockup using a projector-camera system. The specular intensity and position are evaluated in terms of magnitude and inauthenticity. For the specular intensity evaluation, it was found that changes to specular intensity in the CG parameters were equal to the changes in the brightness sensibility of the object viewed. Furthermore, the results of inauthenticity evaluations clarified the limitations of the viewpoint range. The specular appearance from the $60^{\circ}$ viewpoint gave observers the impression that the form and position of the specular reflection were inauthentic. Therefore, it was determined that the control of appearance in our digital mockup was only suitable for observations within the range from $-45^{\circ}$ to $45^{\circ}$.
\end{abstract}

Keywords: Specular appearance, subjective evaluation, projector-camera system, digital mockup.

\section{Introduction}

Research in the field of computer science is advancing steadily towards the realization of seamless integration of the real and virtual worlds [1]. For example, as a result of remarkable progress in the field of computer graphics (CG), it has become possible to accurately reproduce various visual scenes by exploiting the physical properties of the ways in which light interacts with the real world, such as reflection, absorption, and interference [2]. Furthermore, the combination of $\mathrm{CG}$ and computer vision (CV) has paved a way toward developing advanced three dimensional (3D) vision systems that are further erasing borders between actual and virtual reality. 
Because the appearance of computer-generated 3D objects can be controlled easily, 3D reproduction has become increasingly common in various research and industrial applications. In particular, accurate 3D reproduction is very important in a wide range of industrial processes such as design assessment, reverse engineering, and quality inspection. For instance, the visual appearance of physical or virtual objects (such as digital mockups 3]) can be changed easily by controlling specular and diffuse light reflections. Thus, the ability to change the appearance of digital mockups using 3D reproduction techniques can enrich our imaginations.

In general, there are two types of digital mockup: full digital mockups and composite-type digital mockups. Full digital mockups reproduce both the shape and appearance of an object in virtual reality. There are several 3D reproduction methods that use full digital mockups such as holograms, integral photography displays, and light field displays. Unfortunately, employing such methods introduces significant practical challenges 415. In fact, while head-mounted displays or active stereographic 3D shutter glasses might be the simplest ways to reproduce the 3D appearance of a full digital mockup, wearing such glasses can sometimes cause uncomfortable sensations that hinder their practical use [6].

In contrast, composite-type digital mockups address the issues inherent with full digital mockups by reproducing 3D appearance using projection displays and unfinished actual mockups. One advantage of composite-type digital mockups is that they eliminate the need for special glasses, while a disadvantage is that an actual 3D form of the object must be fabricated. Fortunately, recent developments in the field of 3D printing have enabled us to create mockups of almost any shape [7. The images projected onto such mockups are generated by calculating the desired appearance using well-developed CG techniques. The use of projection images can allow reproduction of various composite-type digital mockup views, which allow multiple observers to make observations from different positions and thus produce a thorough evaluation of the image design. However, there are some difficulties related to finding correct settings for CG images, such as reflectance and position, so that they match seamlessly with human visual sensibilities on composite-type digital mockups. In order to achieve an authentic visual appearance, it is important clarify how CG parameter changes correlate with visual sensibility through projection image changes.

Accordingly, this paper presents subjective evaluations made by multiple observers when investigating the relationships between changes in CG parameters and the perceived specular appearance of a composite-type digital mockup. Specular appearance is considered to be the most important appearance attribute, and, during design validation, plays a crucial role in the final decision making process for a variety of products. In specular appearance reproduction, both the intensity and position of the specular reflectance must be set. Specular intensity is controlled by the reflectance coefficient defined by the reflection model used. According to the Weber-Fechner's law, the relationship between the intensity of reflected light and its perceived magnitude is logarithmic [8]. Therefore, it was 


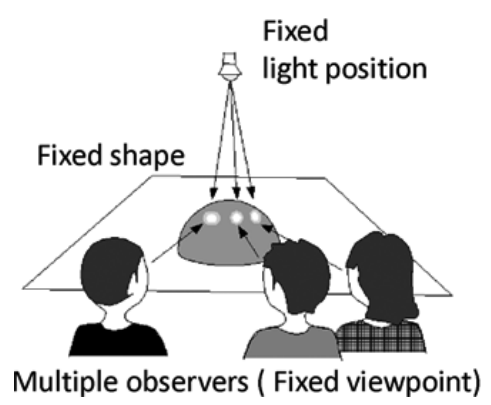

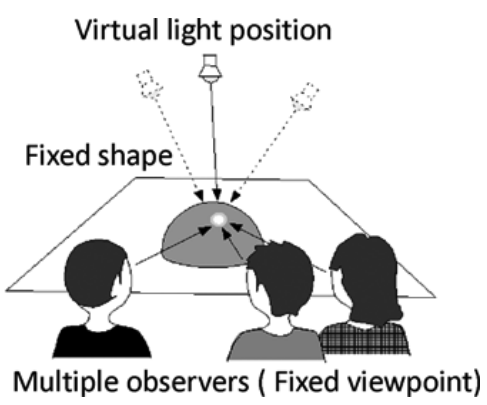

Fig. 1. Illustrated explanation of virtual light positioning for multiple observers (a):Freedom at specular reflection position, (b):Freedom at light position

necessary to determine whether this law is applicable for the CG setting and observer perceptions.

In contrast, in CG settings, the position of a specular reproduction is specified by three parameters, the position of the light source, the shape of the object, and the viewpoint of the observer. Here, a contradiction appears in that each viewpoint is unique, even if the shape and position of the light source are fixed. Two or more specular reflections corresponding to multiple observations may be reproduced on digital mockups, as shown in Fig. 1(a). Note that, during our observations, no negative sensations were experienced, even when a single specular reflection was observed from various viewpoints, as shown in Fig. 1(b). This result infers that single specular reproduction with shape fixation can cause two or more observers to perceive the existence of a virtual light at an arbitrary position. Therefore, for a design assessment system, we determined that it was necessary to examine the viewpoint range of multiple observers.

This paper focuses on the construction of a projector-based reproduction system and subjective evaluations of the relationship between CG parameters and visual sensibility. A brief review of related works is presented in Section 2, Section 3 presents our experimental system, while our rendering method is discussed in Section 4. The results of subjective evaluations of sensibility magnitude and viewpoint range of specular appearance are described in Section 5] and 6 respectively. Finally, in the conclusion, we discuss the evaluation results and the limitations of our system.

\section{Related Works}

The virtual reality production technology that integrates projected images and digital mockups arose simultaneously with development in computers and projectors. The initial form of this technology was simple image transcription, where virtual information was projected onto walls and screens without using actual mockups [910]. The fusion with CV made it possible to precisely measure the position and the shape of an actual $3 \mathrm{D}$ object, which then made possible the 
development of 3D projector-camera systems. Projector-camera systems can produce various image combinations with authentic appearing visual textures [1112]. Other recent research has resulted in improvements to the projection image quality, by taking into consideration the object's surface reflectance and surrounding ambient light [1314].

Still other research efforts have worked to reproduce projection images that include not only texture, but appearance elements such as specular reflectance or shading [1516]. Rasker et al. developed a realistic projection system, as shown in Fig. 2, This work succeeded in producing visual texture and appearance of complex shapes using 3D measurement technology and control method utilizing multiple projectors [17. Kamimigaki et al. have also developed a reproduction system that was able to project specular appearance onto a cylinder mockup, as shown in Fig. 3. This system utilizes high speed rendering to reproduce the desired specular appearance based on the observer's viewpoint [18. Figure 4 shows a flexible system consisting of a multiple projector-camera setup suitable for multiple observers 19 .

As mentioned previously, it is already possible to create actual 3D appearance textures on an object by projecting imagery information that conforms to the shape of mockup. Furthermore, with the integration of CV and CG techniques, it now appears possible to design digital mockups incorporating arbitrary color

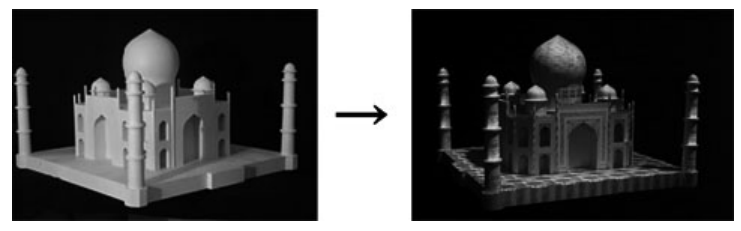

Fig. 2. Realistic visual texture and appearance can be produced using shader lamps to project light information on a $3 \mathrm{D}$ object, as seen in this example
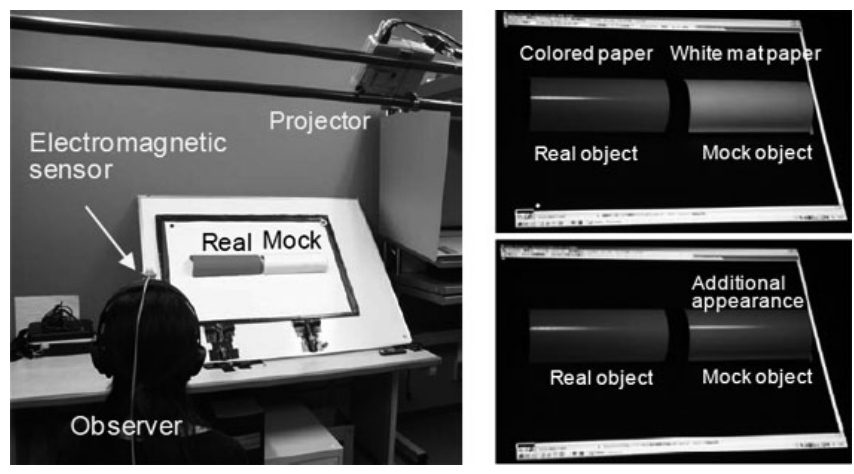

Fig. 3. Experimental system using a projector and head tracking system (a): Experimental arrangement (b): Result of specular appearance reproduction 

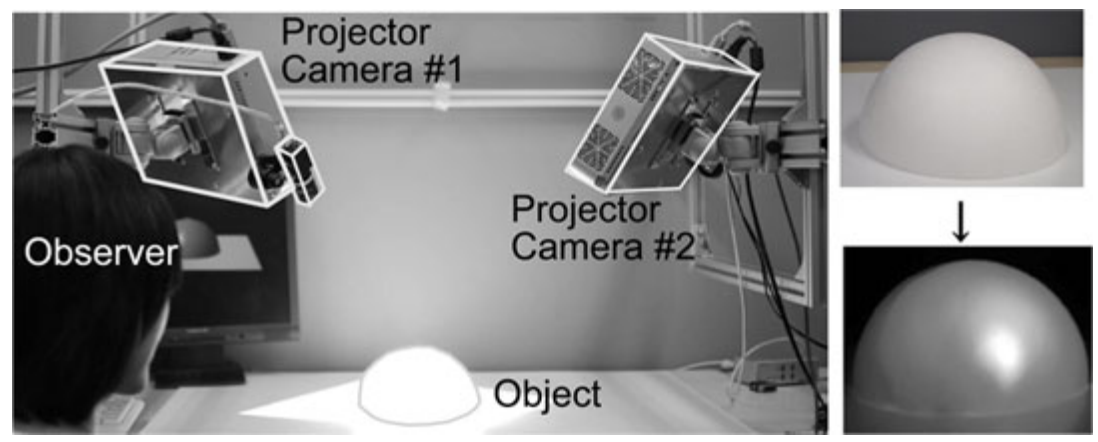

Fig. 4. Prototype system for reproducing actual visual appearance information on a mock $3 \mathrm{D}$ object

(a): Experimental arrangement (b): Result of specular appearance reproduction

and specular reflection. The practical simulation of various appearances is performed by controlling the CG parameters. Unfortunately, no amount of control guarantees that the image produced will satisfy visual sensibility requirements. To create an accurate matching between the virtual and real worlds, it is necessary to clarify the gain and control range necessary to satisfy visual sensibility requirements.

Therefore, in this research, we subjectively evaluated the relationship between reproduction results and visual sensibility using an appearance reproduction system that can be controlled by the projected image. We focused on the visual sensibility as difference is verified about the magnitude and the position of the specular reflectance. Significant attention has been paid to the specular appearance of an image recently, because of its important role in evaluating the quality of materials.

\section{Projector-Camera System}

To project an exact desired appearance on a 3D object, it is first necessary to know the shape of the object and its precise location relative to the projector. For increased system versatility, it is preferable to be able to reproduce such an appearance even the object is moved to another position. Therefore, our projector-camera system enabled to perform an auto geometric calibration.

In the first step, the projector-camera system should correct for any geometrical lens distortion. Next, it is necessary to accurately acquire the positions of both the camera and projector. Lens distortion and focal length are calculated as internal parameters [20]. Position and orientation of the projector and camera are calculated as external parameters. Note that the projector and camera optics are assumed using a pinhole camera model. Each parameter could be obtained using the capture method and calculations developed by S. Zhang et al. 21. In our system, the camera first captures an image of a checkerboard projected via 

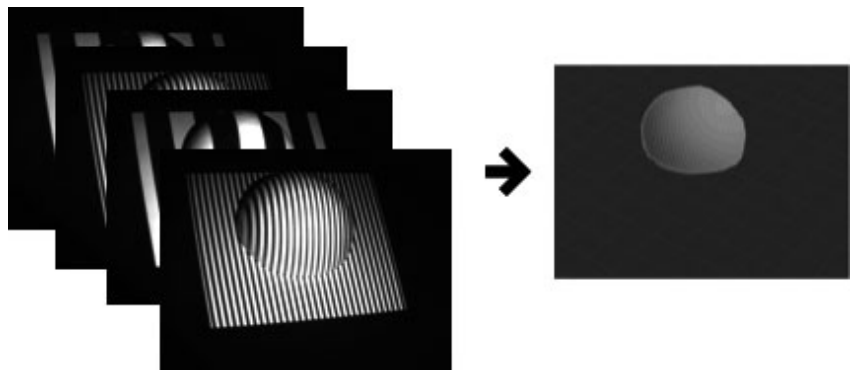

Fig. 5. Shape measurement illustration images

(a): Some structured light on the object (b): Calibrating shape and position of the 3D object

structured light. The calculation then determines the relationship of the corresponding pixels and coordinates using the captured image. Using these obtained parameters, the lens is calibrated and the transformation matrix between the devices is clarified.

After the system is calibrated, the coded structured light method is utilized to measure the position and shape of the mockup. Figure 5(a) shows examples of the images used for shape measurements with the coded structured light method. The images of multiple coded patterns are necessary to construct an accurate model of the shape. Then, based on these images, the 3D position and shape of the mockup is calculated via triangulation, as shown in Fig. 5(b). Calculated 3D data are used as vertex information in CG software. Here, it should be noted that the $3 \mathrm{D}$ data includes the relative position of the mockup at the projected plane. Therefore, accurate image projection onto the mockup becomes possible without misalignment.

\section{Rendering Equation for Specular Appearance}

In this section, we describe the rendering process used to reproduce the desired appearance based on the mockup vertex data. To reproduce a colored mockup with specular reflectance characteristics, a dichromatic reflection model was adopted in our rendering process. Figure 6] shows dichromatic reflection model. In the dichromatic model, incident light is reflected at the surface, while the rest enters the object and is absorbed. In the specular model, when light is reflected at the object's surface, the angle of incidence equals the angle of reflection. Diffuse light is generated by multiple reflections and scattering in the object. There are various dichromatic reflection models including Phong [22, TorranceSparrow [23, and Ward [24]. In our system, Phong's model was adopted due to its low computational costs and few adjustable parameters. Here, reflected light $I_{r}$ without ambient is modeled as follows:

$$
I_{r}=I_{i}\left(k_{d} \cos \alpha+k_{s} \cos ^{n} \beta\right),
$$



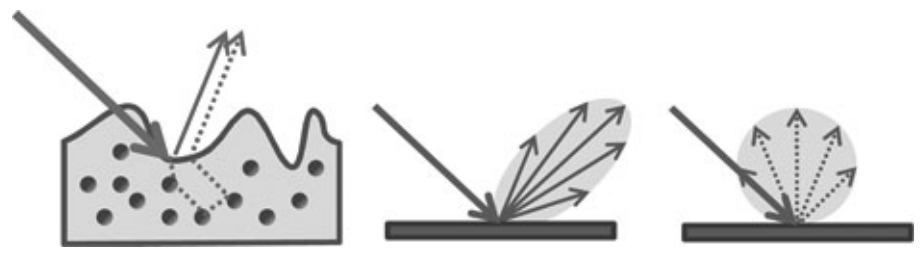

Fig. 6. Reflected light distribution in Phong's Model

(a): Dichromatic reflectance (b): Specular reflectance (c): Diffuse reflectance

where $I_{i}$ denotes incident radiance from the light source, $k_{d}$ is the coefficient of the diffuse reflectance, $k_{s}$ is the coefficient of the specular reflectance, $\alpha$ is the angle between the surface normal of the object and the light source direction, $\beta$ is the angle between the viewpoint and the specular reflection, and $n$ is the specular reflection characteristics. The intensity distribution of reflected light in Phong's model is shown in Fig. 6(a). Specular reflection refers to light that is reflected symmetrically to the normal, as shown in Fig. 6(b). Diffuse reflected light is independent of the incident light direction and viewpoint location because it scatters evenly in all directions, as shown in Fig. 6(c).

Projection image pixels are calculated by Phong's model according to the viewpoint and light source positions. Observers perceive the reflected light from the surface of the digital mockup, which is generated by the projection image. In this experiment, the coefficient of the diffuse reflectance, $k_{d}$ is fixed because the digital mockup color is constant. In contrast, the coefficient of the specular reflectance, $k_{s}$ is a variable parameter used to evaluate the relationship between visual sensitivity and specular intensity, where $n$ is fixed. Furthermore, the position of the light source and viewpoint are also fixed in Phong's model in order to facilitate appearance evaluations from various positions by multiple observers.

\section{Experiment}

Using our projector-camera system, we were able to produce reflected specular light appearance on a mockup and could subjectively evaluate the relationship between the results and visual sensibility. In our experiments, the specular intensity and position generated by each viewpoint were evaluated as the magnitude and authenticity of visual sensibility. Ideally, these evaluations should be performed as separate experiments. However, it was believed that the excessively long evaluation periods required would introduce instability, and thus produce uncertain results. Therefore, we developed a method of conducting these evaluations simultaneously, as described in Chapter 5.1. For the intensity evaluation, observers described their sensibility appraisals based on the magnitude estimation method according to the changes to the specular coefficient $k_{s}$ [25. At the same time, they were asked to complete a questionnaire about the authenticity of the specular reproduction at each intensity and viewpoint. 


\subsection{Experimental Layout}

Figure 7 shows our experimental layout. In this system, a 3LCD type projector with $1024 \times 768$ resolution (Panasonic, PT0LB51NT), and a monochrome CCD camera with $1280 \times 960$ resolution (IMI Tech, IMC-17FT) were used. The mockup was a styrene foam hemisphere placed in the center of the projection area. Furthermore, in this system, the projector-camera system was covered with a blackout curtain because it was considered important that the observer perceive only the appearance projected onto the mockup during the evaluation period. Viewpoints, defined as the observer sitting positions, were set at $0^{\circ}, 15^{\circ}$, $30^{\circ}, 45^{\circ}$, and $60^{\circ}$, respectively. Here, the $0^{\circ}$ position is defined as the location where specular reflection directly faced the observer, as shown in Fig. 8. The distance from the hemisphere to the observer was set at 2.5 meters, at which point the patterned indented surface of the styrene foam was not visible. At each viewpoint, we recorded the observer's answers regarding magnitude and any comments about the specular image superimposed on the mockup.

It is understood that, for a digital mockup, limitations in projector brightness will lead to specular intensity limitations. As a result of a preliminary experiment in our system, we found that the practical intensity of the digital mockup saturated at $k_{s}=0.4$. Therefore, the specular coefficient $k_{s}$ was set at $0.1,0.2,0.3$, and 0.4, respectively, as shown in Fig. 9. The intensity of the perceived specular reflection was evaluated using the magnitude estimation method. This method
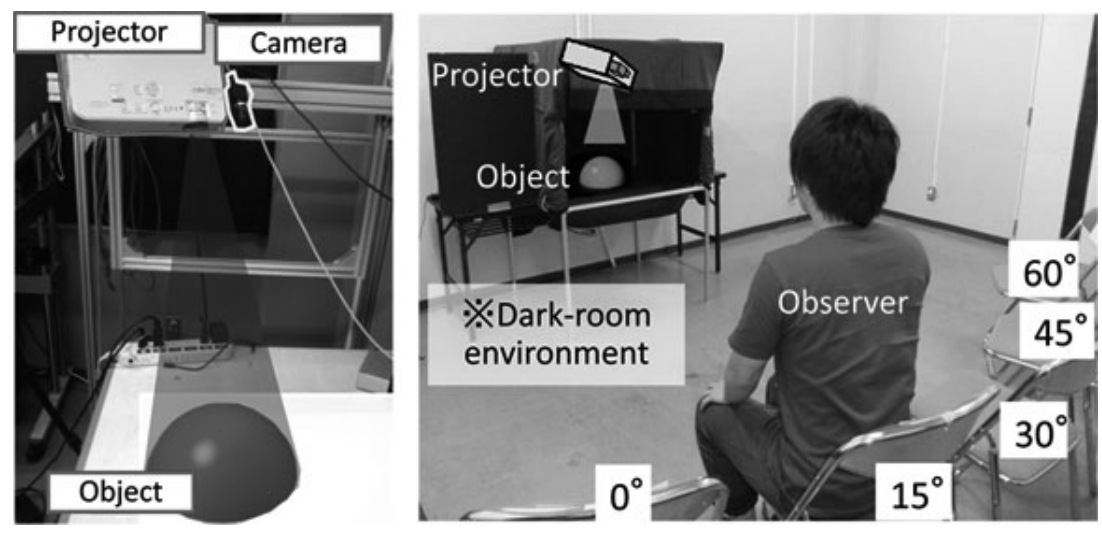

Fig. 7. Experimental layout

(a): Projector-camera system (b): Viewpoints for subjective evaluation
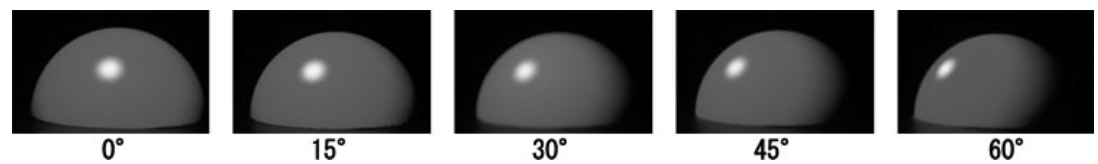

Fig. 8. Specular appearance at each viewing position 


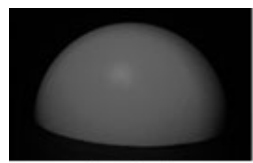

$k s=0.1$

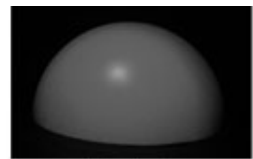

$k s=0.2$

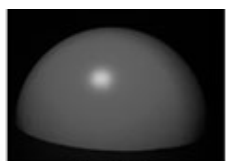

$k s=0.3$

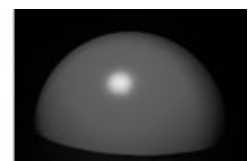

$k s=0.4$

Fig. 9. Specular appearance for each coefficient $k_{s}$

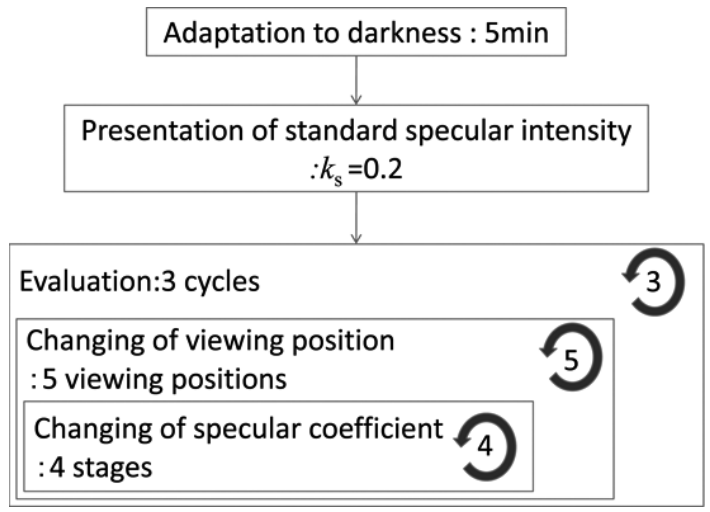

Fig. 10. Flow of experimental process

makes it possible to determine the sensitivity of an observer as a numerical value. Based on our arbitrary definition that a standard specular intensity at $k_{s}=0.2$ would receive a score of 100 , observers were asked to evaluate and record scores for various specular reflection intensities displayed at random. Some other limitations were set as experimental conditions. For example, observers were asked to make their evaluations while moving their heads as little as possible and to answer intuitively. However, we did not limit observation time. Under these conditions, 10 men and women in their twenties participated in the experiment.

The experimental process flow is shown in Fig. 10, After five minutes of dark adaptation, observers were asked to observe and evaluate the specular intensity of the standard appearance at $0^{\circ}$ viewpoint. Then, they experienced intensity changes in four stages and were moved among five positions, while providing their evaluations at each change. A total of three experiment cycles were performed for each observer.

\section{Subjective Evaluation}

\subsection{Intensity of Specular Appearance Using Magnitude Estimation Method}

Typical subjective evaluation results are shown in Fig. 11. The x-axis indicates the luminance at the center of the specular reflection appearance, while the $y$-axis 

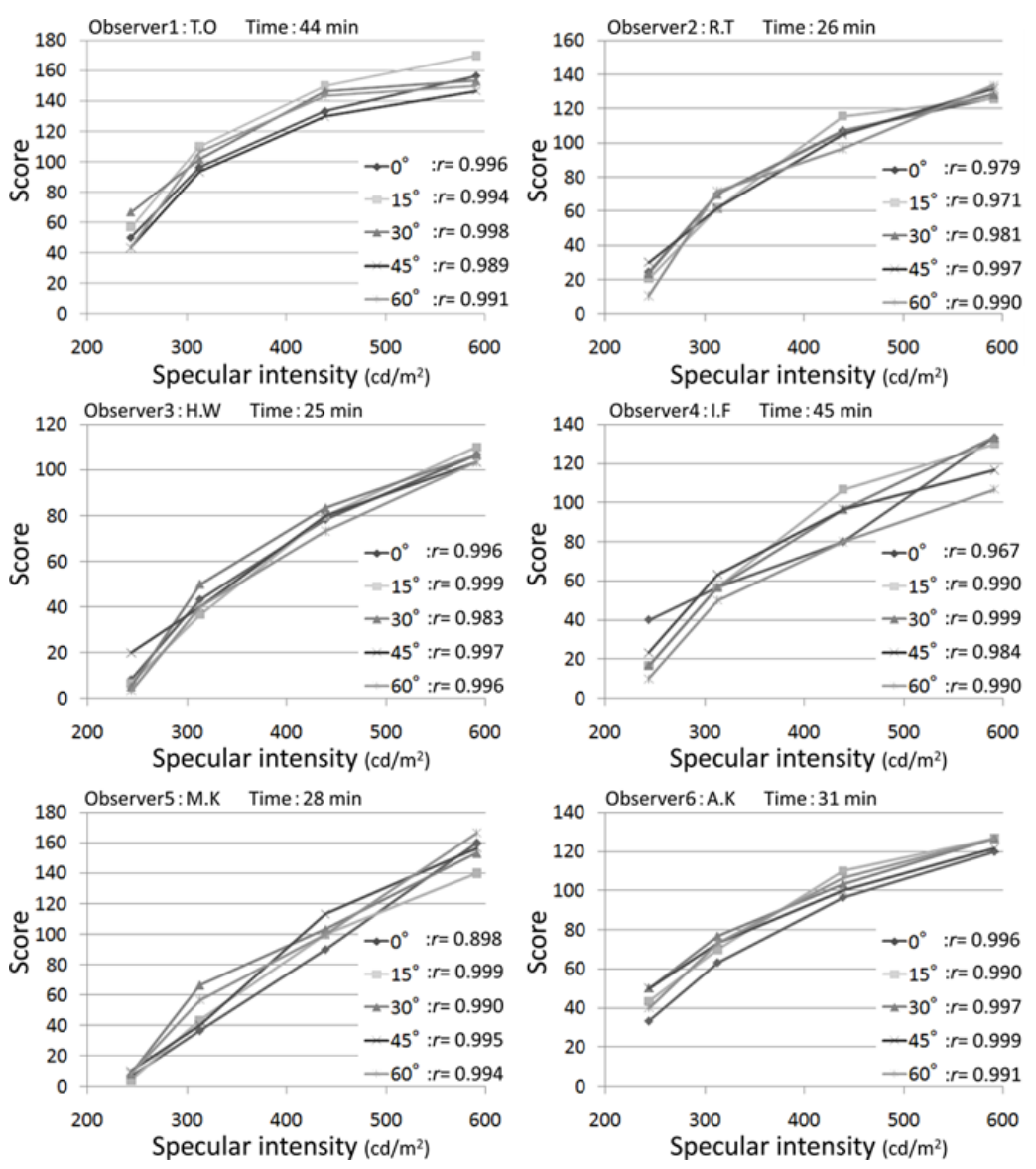

Fig. 11. Magnitude estimation results

The $\mathrm{x}$-axis is the luminance at the center of specular appearance while the $\mathrm{y}$-axis is the subjective evaluation score for specular intensity.

indicates the subjective evaluation score for specular intensity recorded using the magnitude estimation method. The values of $r$ in Fig. 11 indicate correlation coefficient with a logarithm at each viewpoint. The luminance correspond to each specular reflectance coefficients of $k_{s}=0.1,0.2,0.3,0.4$, as shown in Table 1.

The subjective evaluation results showed good correlation with a logarithm, and it was clear that the specular reflection intensity of our system followed Weber-Fechner's law. Additionally, it was determined that the evaluation score for the specular appearance intensity did not differ at each viewpoint. Thus, with the projector-camera system, we could conclude that visual sensitivity reported for specular intensity changes were similar to actual brightness variations that occur when coefficient $k_{s}$ was changed. 


\subsection{Verification of Inauthenticity Due to Changes in Viewing Position}

Figure 12 shows the number of observers that reported ginauthenticity" at each viewpoint. The $\mathrm{x}$-axis indicates the specular intensity $k_{s}$, while the $\mathrm{y}$-axis indicates the viewpoint, the $\mathrm{z}$-axis indicates the numbers of observers that reported inauthenticity two or more times during their three experiment cycles.

Numerous inauthenticity reports were received in the case of $k_{s}=0.1$ and $k_{s}=0.4$, and inauthenticity was also reported for all cases of the $k_{s}$ at $60^{\circ}$ viewpoint. The reason given by most observers at the $k_{s}=0.1$ scenes, was that the specular reflection looked artificial because its specular intensity was too weak. When explaining their inauthenticity report at $k_{s}=0.4$, most observers commented that the specular intensity was quite different from reality because the specular intensity was too strong.

In contrast, the specular appearance from the $60^{\circ}$ viewpoint gave observers a different impression of inauthenticity, with many comments that the form and position of the specular reflection were strange. Such observers said that the

Table 1. Luminance at the specular reflection center

\begin{tabular}{c|c}
\hline$k_{s}$ & Luminance: $\mathrm{Y}\left(c d / m^{2}\right)$ \\
\hline 0.1 & 224 \\
0.2 & 313 \\
0.3 & 439 \\
0.4 & 591 \\
\hline
\end{tabular}

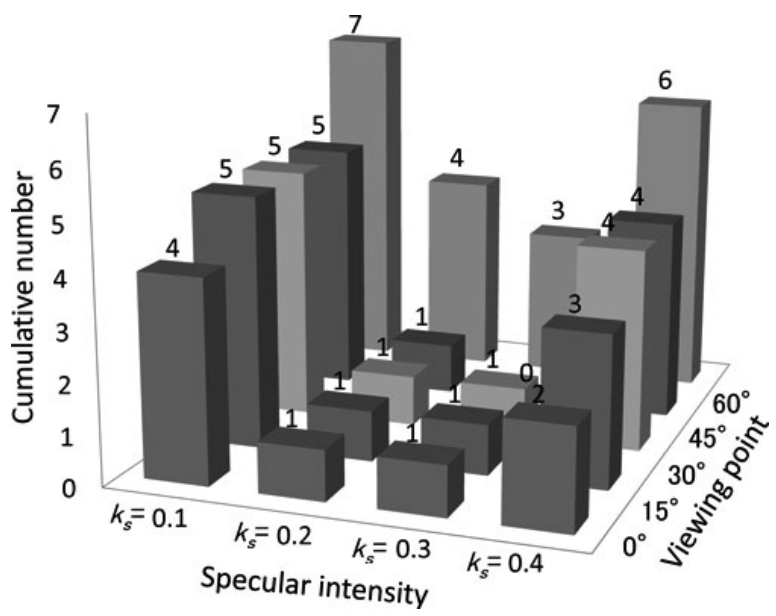

Fig. 12. Evaluation results for inauthenticity

The $\mathrm{x}$-axis is the specular intensity $k_{s}$, the $\mathrm{y}$-axis is the viewpoint, and the $\mathrm{z}$-axis is the number of observers that reported inauthenticity two or more times during their three experiment cycles. 
specular shape was strangely circular and that the specular position was too low. Based on the above-mentioned comments, we concluded that in our system, specular reproduction can only provide authentic sensibility within the range of $0^{\circ}$ to $45^{\circ}$.

\section{Conclusion}

This paper presented subjective evaluations of multiple observers in order to investigate the relationship between changes in CG parameters and visual sensibility. Using a composite-type digital mockup system, the specular appearance of a CG generated image was reproduced on a mockup using a projector-camera system. The pixels of the projection image were calculated using Phong's model based on the viewpoint position and light source. In our experiment, the specular coefficients $k_{s}$ in Phong's model were set at $0.1,0.2,0.3$, and 0.4 , and the specular reflection position was fixed. Observer viewpoints were sitting positions set at $0^{\circ}, 15^{\circ}, 30^{\circ}, 45^{\circ}$, and $60^{\circ}$. The specular intensity and position generated at each viewpoint were evaluated using the magnitude estimation method and authenticity of visual sensibility was determined. Evaluation results of specular intensity clarified that the specular reflection intensity of our system followed Weber-Fechner's law. Additionally, it was found that the intensity evaluation score for specular appearance did not differ by viewpoint. Therefore, for the specular intensity evaluation, it was determined that the change in specular intensity in the CG parameter was equal to the change in the sense of brightness, regardless of the viewpoint. An evaluation of the sense of inauthenticity experienced clarified limitations within the viewpoint range. In particular, many observers reported feelings of inauthenticity at the $60^{\circ}$ viewpoint. Therefore, in our system, we concluded that $\mathrm{CG}$ image settings such as reflectance and position only corresponded accurately to visual sensibility from $-45^{\circ}$ to $45^{\circ}$.

In the future, it is believed that using projector-camera systems, 3D reproduction will become an increasingly common tool in various research and industrial applications because it allows the appearance attributes of computer-generated $3 \mathrm{D}$ objects to be controlled easily.

\section{Limitation and Future Work}

Our experiments focused on a simple hemispherical shape, which simplified evaluations into the relationship between $\mathrm{CG}$ parameters and visual sensibility. However, practical digital mockups will require reproducing the appearance of complex shapes. Therefore, it will be necessary to verify the clarification results for each different mockup shape. Furthermore, reports of inauthentic specular appearances were obtained via a questionnaire, even though specular intensity was evaluated on a numerical scale. Therefore, it is not clarified of the magnitude of inauthenticity. The quantitative evaluation will be examined to the inauthenticity observation in the future. 
As part of our future developments, we intend to improve the practical application and expansion of digital mockups so that rendering techniques and device settings can be revised to reproduce various appearances other than specular reflections. Furthermore, to improve the practical utility of the system, will be necessary to enable it to be used for close-up observations, which will require higher levels of reproduction accuracy in terms of image detail, grain, etc. Accordingly, we expect to focus on the development of advance reproduction techniques that can render high resolution images and bump mapping.

\section{Acknowledgements}

Norimichi Tsumura is partly supported by JSPS Grants-in-Aid for Scientific Research (19360026). Shoji Yamamoto and Norimichi Tsumura are also partly supported by JSPS Grants-in-Aid for Scientific Research (20500198).

\section{References}

1. Weiser, M.: The computer for the 21st century. Scientific American 272(3), 78-89 (1995)

2. Foley, J.: Computer graphics: principles and practice. Addison-Wesley Professional, Reading (1995)

3. Beyer, H., Holtzblatt, K.: Contextual design. Interactions 6(1), 32-42 (1999)

4. Pastoor, S., Wöpking, M.: 3-D displays: A review of current technologies. Displays $17(2), 100-110$ (1997)

5. Jones, A., McDowall, I., Yamada, H., Bolas, M., Debevec, P.: Rendering for an interactive 360 light field display. In: ACM SIGGRAPH 2007 Emerging Technologies, p. 13. ACM, New York (2007)

6. Lantz, E.: The future of virtual reality: head mounted displays versus spatially immersive displays (panel). In: Proceedings of the 23rd Annual Conference on Computer Graphics and Interactive Techniques, pp. 485-486. ACM, New York (1996)

7. Hiller, J., Lipson, H.: Tunable digital material properties for $3 \mathrm{D}$ voxel printers. Rapid Prototyping Journal 16(4), 241-247 (2010)

8. Fechner, G., Adler, H., Howes, D., Boring, E.: Elements of psychophysics (1966)

9. Raskar, R., Welch, G., Cutts, M., Lake, A., Stesin, L., Fuchs, H.: The office of the future: A unified approach to image-based modeling and spatially immersive displays. In: Proceedings of the 25th Annual Conference on Computer Graphics and Interactive Techniques, pp. 179-188. ACM, New York (1998)

10. Kruger, W., Bohn, C., Frohlich, B., Schuth, H., Strauss, W., Wesche, G.: The responsive workbench: A virtual work environment. Computer 28(7), 42-48 (2002)

11. Pinhanez, C., Kjeldsen, R., Levas, A., Pingali, G., Podlaseck, M., Sukaviriya, N.: Applications of steerable projector-camera systems. In: Proceedings of the IEEE International Workshop on Projector-Camera Systems at ICCV 2003 (2003), Citeseer

12. Yoshida, T., Horii, C., Sato, K.: A virtual color reconstruction system for real heritage with light projection. In: Proceedings of VSMM, pp. 161-168 (2003) 
13. Grossberg, M., Peri, H., Nayar, S., Belhumeur, P.: Making one object look like another: Controlling appearance using a projector-camera system. In: Proceedings of the IEEE Computer Society Conference on Computer Vision and Pattern Recognition, CVPR 2004, vol. 1, IEEE, Los Alamitos (2004)

14. Amano, T., Kato, H.: Appearance control by projector camera feedback for visually impaired. In: 2010 IEEE Computer Society Conference on Computer Vision and Pattern Recognition Workshops (CVPRW), pp. 57-63. IEEE, Los Alamitos (2010)

15. Mukaigawa, Y., Nishiyama, M., Shakunaga, T.: Realization of virtual photometric environment by photometric pattern projection. In: Proceedings of the IEEE International Symposium on Computational Intelligence in Robotics and Automation, 2003, vol. 1, pp. 435-440. IEEE, Los Alamitos (2003)

16. Konieczny, J., Meyer, G.: Material and color design using projectors. In: CGIV 2006: Third European Conference on Colour in Graphics, Imaging, and Vision, pp. 438-442. Citeseer (2006)

17. Raskar, R., Welch, G., Low, K., Bandyopadhyay, D.: Shader lamps: Animating real objects with image-based illumination. In: Rendering Techniques 2001: Proceedings of the Eurographics Workshop, London, United Kingdom, June 25-27, p. 89. Springer, Wien (2001)

18. Yamamoto, S., Tsuruse, M., Takase, K., Tsumura, N., Nakaguchi, T., Miyake, Y.: Real-Time Control of Appearance on the Object by using High Luminance PC Projector and Graphics Hardware. In: The 13th Color Imaging Conference, Scottsdale, USA (2005)

19. Kamimigaki, S., Yamamoto, S., Tsumura, N., Nakaguchi, T., Miyake, Y.: Real reproducing of $3 \mathrm{D}$ appearance with multi-projectors and cameras. In: The 17th Color Imaging Conference (2009)

20. Zhang, Z.: Flexible camera calibration by viewing a plane from unknown orientations. In: ICCV, p. 666. IEEE Computer Society, Los Alamitos (1999)

21. Zhang, S., Huang, P.: Novel method for structured light system calibration. Optical Engineering 45, 083601 (2006)

22. Phong, B.: Illumination for computer generated pictures. Communications of the ACM 18(6), 311-317 (1975)

23. Torrance, K., Sparrow, E.: Theory for off-specular reflection from roughened surfaces. Journal of the Optical Society of America 57(9), 1105-1114 (1967)

24. Ward, G.: Measuring and modeling anisotropic reflection. In: Proceedings of the 19th Annual Conference on Computer Graphics and Interactive Techniques, pp. 265-272. ACM, New York (1992)

25. Torgerson, W., Social Science Research Council (US). Committee on Scaling Theory and Methods: Theory and methods of scaling, vol. 1967. Wiley, New York (1958) 